\title{
Eye gaze estimation from a video
}

\author{
Djamel Merad* \\ LIRIS UMR-5205 Laboratory
}

\author{
Stéphanie Metz ${ }^{\dagger}$ \\ ICAR UMR-5191 Laboratory
}

\author{
Serge Miguet \\ LIRIS UMR-5205 Laboratory
}

\begin{abstract}
Our work focuses on the interdisciplinary field of detailed analysis of behaviors exhibited by individuals during sessions of distributed collaboration. With a particular focus on ergonomics, we propose new mechanisms to be integrated into existing tools to enable increased productivity in distributed learning and working. Our technique is to record ocular movements (eye tracking) to analyze various scenarios of distributed collaboration in the context of computer-based training. In this article, we present a low-cost oculometric device that is capable of making ocular measurements without interfering with the natural behavior of the subject. We expect that this device could be employed anywhere that a natural, non-intrusive method of observation is required, and its low-cost permits it to be readily integrated into existing popular tools, particularly E-learning campus.
\end{abstract}

CR Categories: I.4.m [Image Processing And Computer Vision]: Miscellaneous-; I.5.m [Pattern Recognition]: Miscellaneous

Keywords: Visual direction estimation, Head tracking.

\section{Introduction}

The principle of the oculometry is to measure the position and orientation of the eye of a subject as he looks at a computer monitor. A software program, which knows what is displayed on the monitor at what particular instant, is able to determine what the subject had been looking at for any specified instant. For many years, this technique has been used by psychologists to study behavioral responses to images, but more recently, it has spread other domains such as that of ergonomicists and Web masters. [?] provides an excellent survey of eye tracking applications in several information processing tasks. In the case of analysis of the relationship between image observation and cognitive response, we can quote for example: the inspection of visual scenes [Henderson and Hollingworth 1998], visual Inspection [Reingold et al. 2002], industrial engineering and human factors [Anders 2001]. But in the last few years, this technique has been applied to determine the usability of Web, and to determine why some web sites are more effective than others [Sibert and Jacob 2000]. This is a direct consequence of the development of information technologies and communication.

Our work will relate to find precise oculometric indicators that manifest during distributed computer based training. We prescribe a

\footnotetext{
*e-mail: djamel.merad@liris.cnrs.fr

†e-mail: stephanie.maille@univ-lyon2.fr

‡e-mail: serge.miguet@liris.cnrs.fr
}

control environment where two geographically dispersed interlocutors engaged in collaborative work via face-to-face video conferencing. Each interactive station has a camera that captures images of the subject's faces and eyes as movements occur and words are spoken. As the collaboration progresses, we collect indicators which will allow us to know, for each instant, what is consulted by the interlocutors and for what type of interactions. Thus, it will be necessary to synchronize the verbal interactions with the glances of the subjects at each instant of collaborative activity.

Previous work [Corbel et al. 2003], [Baker et al. 2002] have made it possible to enumerate the types of interactions observed at the instances during collaboration using tools that allow the mutual construction of a common conceptual representation of the subject matter being discussed by the collaborators. In addition, tools that facilitate this type of activity have been developed. In particular, DREW makes it possible to collaborate and jointly synthesize knowledge through primitives such as the catalogue, white board, a text tool, an argumentative graph. These various studies are based on the analysis of verbal corpora (catalogue) and argumentative graphs. They do not make it possible to pin-point the specific events that initiate an interaction for mutual knowledge dissemination. Indeed, the interlocutor does not always refer verbally to the information which enabled him to find such solutions. So if the ultimate goal is to help the interlocutors in their collaboration, it is necessary to characterize the chronological process of mutual transfer and assembly of knowledge. For example, if one notes by oculometry that an interlocutor seeks information on the screen to argue, it would seem most appropriate that assistive action would immediately present this information centrally on the screen (figure 1).

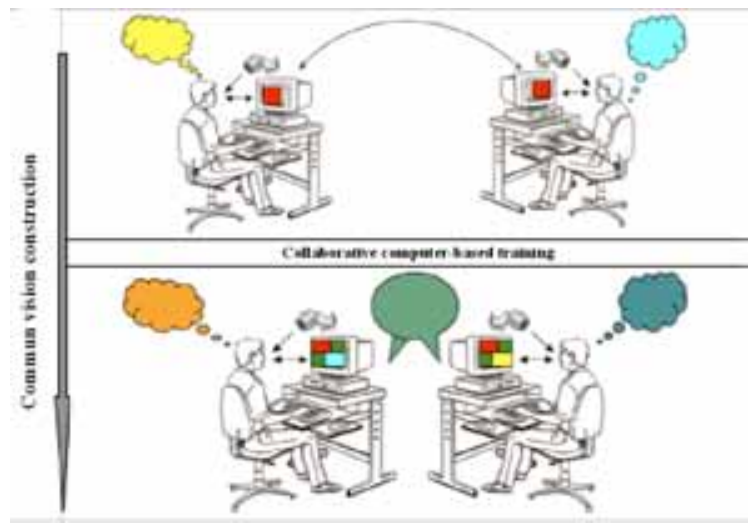

Figure 1: collaborative task

Several techniques to determine what assistive action to take during the follow-up of a glance exist. During last decades, these techniques gained much in precision, which makes their fields of application very broad. On the other hand, no instrument can be perfect in a situation where none satisfies all the requirements.

We can classify the techniques currently developed in three groups, according to the degree of contact with subject [Collewijn 1999], [Glenstrup and Nielson 1995].

- Techniques based on the reflection of a luminous ray on the cornea. 
- Techniques based on the application of a special contact lens.

- Techniques of electro-oculography.

We point out that tools based on corneal oculometric reflection are the most used. These techniques are those which present more comfort to the subjects, do not require the presence of specialists (ophtalmologist) and, under good experimental conditions, give precise results. We find on the market two types of systems using corneal reflection: oculometer with helmet (camera placed on the head) and oculometer without helmet (camera placed on the monitor). The oculometer with helmet is largely used because it is adaptable and usable in several situations (reading on dashboard, reading on monitor, subject moving...). For this reason, practically all the companies have developed a model whose general principle is the same. However for our purposes, we will not be able to use this apparatus because the helmet is not comfortable and will obviously interfere with the subject.

The oculometer without helmet makes it possible to track in real time the movements of the eye using a light-emitting diode (LED) coupled with camera device placed under the monitor. This tool is especially adapted to work on computer displays. The first apparatus of this type uses a head-rest to limit the field of movement of this one. This constraint makes difficult its use for long-lasting experiments, as it results in muscle fatigue. More recently we have seen passive devices that make no contact with the subject.

After seeing several demonstrations of these devices, we have concluded that the most successful is Tobii ${ }^{1}$. This apparatus has the advantage of being fast and easy to use and, more importantly, does not place significant restrictions on the movements of the subject during collaboration. However it is cost-prohibitive, limiting its use as a general-purpose tool.

In short, several kinds of commercial products exist. These products are generally highly accurate and reliable; however all require either expensive hardware or artificial environments (cameras mounted on a helmet, infrared lighting, etc). The discomfort and the restriction of the motion affects the person's behaviour, which therefore makes it difficult to measure his/her natural behaviour. These limitations make them unsuitable for our purpose. We have sought to create a device that removes these limitations.

\section{Proposed Method}

The objective of our study is to correlate the mental representation of exchanged information with the verbal interactions that occur as the representation is built. The oculometry must give indications on the representations of each instant, and show the step-by-step progression of development of the representation as the collaboration proceeds.

First, we analyzed the most popular oculometric. We noted the difficult problems to which they are faced and that still have not been solved in a satisfactory manner. We established that in the best conditions, the system to develop must submit to the following requirements:

- No not hinder the head (or the body) movement.

- Not requires a direct contact with the user: nor helmet, nor lentils.

- An affordable price.

- Good eye gaze localization.

\footnotetext{
${ }^{1}$ http://www.tobii.com
}

- Enable a large vision field.

- Real time response.

- To be easy to use on a large palette of users.

- To be easily usable by the scientist and the user.

To solve this problem, much research has been directed to nonintrusive gaze tracking in the past few years [Wu et al. 2005], [Wang et al. 2003], [Zhu and Yang 2002], [Matsumoto and Zelinsky 2000b], [Pastoor et al. 1999], [Kim and Ramakrishna 1999], [Toyama 1998], [Stiefelhagen and Yang 1997], [Pomplun et al. 1994], [Baluja and Pomerleau 1994]. These systems fall into two categories: analytical approaches and neural network approaches. A neural network based approach takes eye images as input and learns patterns from examples. The reason why they can achieve a higher accuracy is that they take advantage of the pixel intensity information of the whole eye image. Although neural network systems have achieved higher accuracy in offline evaluations, few systems have been applied to real applications, because the trained neural network is too sensitive to changes in users, lighting conditions, and even changes within the user.

An analytical gaze estimation algorithm employs the estimation of the visual direction directly from the eye features such as irises, eye corners, eyelids, etc to compute a gaze direction. If the positions of any two points of the nodal point, the fovea, the eyeball center and the pupil center can be estimated, the visual direction is determined. However, those points are hidden in eyeball thus they will not be viewable without some special equipment. The approach to solve this problem is to estimate the eyeball center from other viewable facial features indirectly. These methods assume that the eyeball is a sphere and the distances from the eyeball center to the two eye corners is known (generally fixed to $13 \mathrm{~mm}$ ). The eye corners are located (for example by using a binocular stereo system) and are then used to determine the eyeball center. They also assume that the iris boundaries show circles in the image and use Hough Transformation to detect them. The center of the circular iris boundary is used as the pupil center.

The drawback of these methods is the fact that the eye model used is simplistic and is not adapted to all persons. It is easy to see that the eyeball form is not a sphere and it's different for each person, also for several pathological reasons (myopia, longsightedness, ...), the optic axis doesn't pass by the eyeball center. In this kind of methods, the accurate determination of the eyeball center is very important. For example, if the eyeball center is detected at $1 \mathrm{~mm}$ from the real position assuming that the eye radius is $13 \mathrm{~mm}$, we will find on a screen placed to $1 \mathrm{~m}$, an errors of $76.9 \mathrm{~mm}$ (figure 2).

Another drawback of this approach is the fact that the accuracy of gaze tracking is greatly depends upon the detection of the eye corners. For some people the detection of corners is not made correctly because of the eyelid form. Another difficult problem is the fact that on particular head positions (with regard to camera), the internal corner of the eye is occluded by the nose, therefore eye gaze direction is not determined.

For these reasons, we proposed to develop an oculometer by a stereoscopic vision system without eye corners detection presented in the figure. We determine the visual direction by estimating the $3 D$ position of the pupil center named $P$ by stereovision triangulation and the eyeball center named $C$ by an oculometric calibration procedure exposed in section 2.1. We assume that when changing the eye gaze, the eyeball rotates around its center and this center is fixed with regard to the head (figure 3).

The principle of this device presented in the figure 4 consists first 


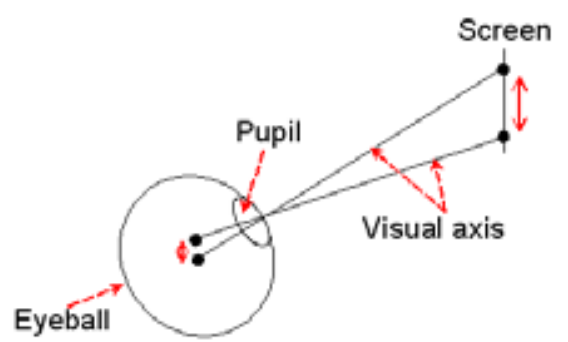

Figure 2: Error propagation

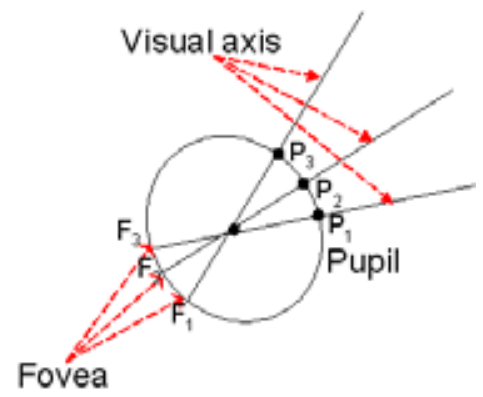

Figure 3: Assumption

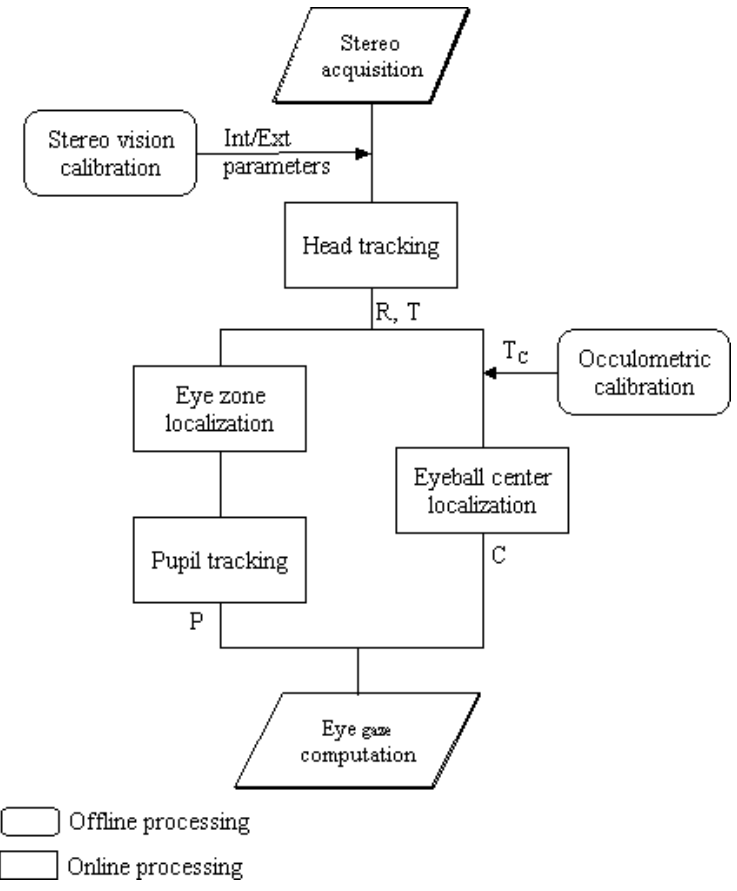

Figure 4: Proposed Method

Collinearity constraints:

$$
\begin{aligned}
& C_{1}=k_{1}\left(P_{1}-Q\right)+Q \\
& C_{2}=k_{2}\left(P_{2}-Q\right)+Q
\end{aligned}
$$

We have also the following transformations:

$$
\begin{aligned}
& C_{1}=R_{1} * T_{c}+T_{1} \\
& C_{2}=R_{2} * T_{c}+T_{2}
\end{aligned}
$$

We obtain an over-determined system with 12 equations and 11 unknown that we solve by the least square method. Theoretically, we need only 1 point and 2 head positions to localise the eyeball center, but in practice and in order to improve the accuracy we should take several points (or head positions).

\subsection{Head tracking}

Many methods enabling real-time object pose computation in relation to the camera exist in the literature. In the approach used for this application, the intrinsic camera parameters are assumed to be known. In addition, the method requires finding the correct matching between a set of $3 \mathrm{D}$ scene points and a set of $2 \mathrm{D}$ image points. Thus, image features must be extracted.

Depending on the objective to reach and the processing to apply on them, image features may be of a very different nature. We can distinguish two main families of image feature extraction approaches:

- approaches which are based on searching a known target in the scene,

- approaches which look for specific geometrical primitives on the object to track.
The user moves the head and stare at the same point, we determine the second world/head transformation $R_{2} T_{2}$ and we compute the second 3D pupil position $P_{2}$. We get the following equations : 


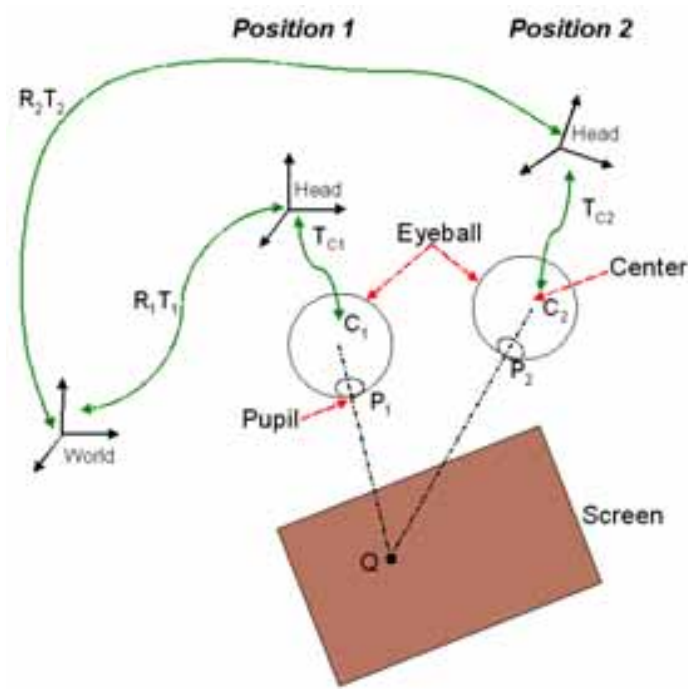

Figure 5: Oculometric calibration

Because of the hard constraint on computation time in our context, we choose the first type of methods. Indeed, instead of trying to recognize an object of the real world, we prefer to detect the targets positioned on it. Image processing algorithms can be optimized for the detection of these targets of known geometry. In addition, coded targets may contain specific features which enable finding the semantic relationship between observed objects and the a priori knowledge about the real world.

Coded targets are widely used in augmented reality systems. One of pioneering systems is the so-called Matrix system proposed by Rekimoto [Rekimoto 1998], renamed later Cybercode [Rekimoto and Ayatsuka 2000]. ARToolkit [Kato et al. 2000], [Kato and Billinghurst 1999] is a library which enables rapidly creating augmented reality applications. Unfortunately, performances of this freeware system ${ }^{2}$ have to be improved. For this reason, research laboratories have developed their own applications as ARTag [Fiala 2005] which look like ARToolkit much. InterSense society [Naimark and Foxlin 2002] has also developed its system coded target based system. The later uses circular targets instead of square targets generally used in prior systems. This system was not the first one ([Cho and Neumann 1998] developed a similar one in 1998) but it is considered as the most performing.

Our goal is to rapidly and accurately detect and localize the user head. That is why we opted for a square target based system.

The processes used to achieve head detection and localization are the following:

- contrast enhancement,

- image binarization,

- connex components analysis for black image area detection,

- high curvature points detection,

- point index reading using white points of the target corners,

- matching target corners which have the same index,

- head pose computation.

${ }^{2}$ http://www.hitl.washington.edu/artoolkit/
Some results are represented in the figure 6, where the head landmark and the eye region are superimposed in the image.
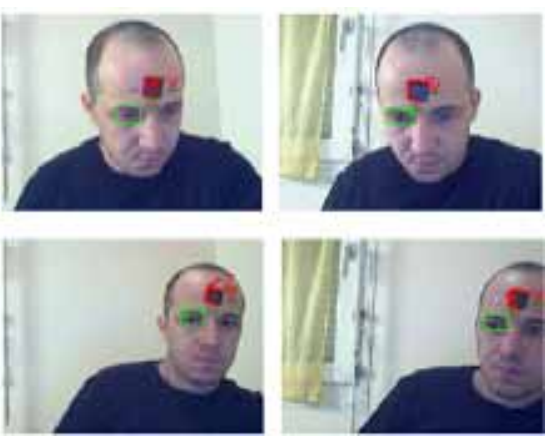

Figure 6: Head and eye tracking

\subsection{Pupil tracking}

The eye gaze determination algorithm cited above assumes that the iris boundaries show circles in the image and used Hough Transformation to detect them (after edge detection). The center of the circular iris boundary was used as the pupil center.

We have tried to use the same procedure and we got some bad results, therefore we can state that this approach has several drawbacks:

- In a natural behavior the eye is naturally mid closed, it is then more difficult to detect a circle from iris edge.

- Eye images available for gaze tracking are typically small in size. Thus the number of available iris edge points is usually very limited, therefore the vote procedure of Hough transform is not appropriated.

- The diameter of an iris is often assumed to be known, while it obviously varies from person to person, and varies when the distance between the user and the camera changes.

- For a large range of gaze directions, the iris in an image cannot be well approximated by a circle (it is an ellipse), other authors use Hough transform to approximate the ellipse but we think that the previous problems still remain.

To detect and localise accurately the pupil in the image, we start by some image processing. We enhance the contrast contour and smooth the homogenous regions by an anisotropic diffusion algorithm [Perona and Malik 1990]. Because of the high contrast between the iris and the eye white, the eye image is easily binarised based on a threshold. Afterwards, we search the connected objects in the binarised image and compute its centroid. To refine this detection, we can use the epipolar constraint and finally, by triangulation we compute the $3 D$ pupil position. Figure 7 shows respectively: original frame, eye zone image and its segmentation and finally the pupil localisation. In this example, hough transform is not able to localise the iris.

\section{Experimental Results}

In order to test the performance of our algorithm for visual direction estimation in a real-time gaze tracking system, we have made some experiments using several real face image sequences. Also, 


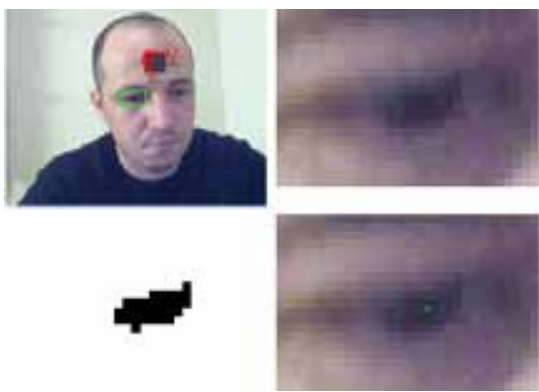

Figure 7: Pupil Tracking

to investigate the accuracy, we have made experiments in a known environment.

There were four markers placed on the four corners on the screen. We take the $3 D$ coordinates of the four markers as the reference coordinates for the true point-of-regard. The errors of the algorithm are computed by comparing the estimated point-of-regard and the reference coordinates. Video cameras were placed at the top of the screen and input image size was $320 \times 240$ pixels. The person sits $0.65 \mathrm{~m}$ away from the camera pair; in this configuration the user has enough space for free movement. The horizontal distance between markers was $29 \mathrm{~cm}$ and the vertical distance was $22.5 \mathrm{~cm}$ (screen 14in). We ask the subject to stare at a marker on a desktop screen using his gaze while moving the head (rotation, translation).

Figure 8 shows some images obtained in a real-time gaze detection experiment. The $3 D$ gaze vectors are superimposed on the tracking result, the low-right image represent a degenerated case. The whole process including face tracking and gaze detection takes about 83 $\mathrm{ms}$, thus the $3 \mathrm{D}$ point-of-regard can be determined at $13 \mathrm{~Hz}$.

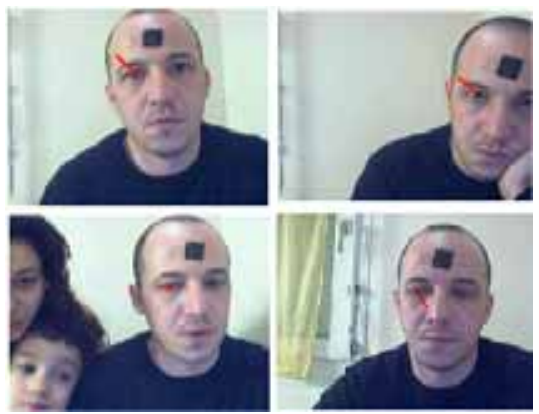

Figure 8: Eye gaze detection

Errors of these algorithms performance on four different sequences are listed in figure 9, figure 10, figure 11, figure 12 corresponding respectively to markers 1 to 4 . We can see the average error of point-of-regard is less than $3 \mathrm{~cm}$ for the Marker 1; this marker is used in oculometric calibration process. The second upper marker the average error of point-of-regard is less than $4.2 \mathrm{~cm}$ and the two lower markers the average errors are less than $5.9 \mathrm{~cm}$. However, we did not take into account the degenerated cases (Marker $1 \approx 14 \%$, Marker $\approx 18 \%$, Marker3 $\approx 23 \%$, Marker4 $\approx 21 \%$,). Indeed, during the tracking process, our algorithm sometimes loses the head or the pupil and in those cases, we get outliers.

We also noticed that in our method as in other analytical approaches, the accuracy of gaze tracking greatly depends upon the resolution of the eye images. Suppose that the size of an eye image is $35 \times 25$, which is a situation when the person is from $65 \mathrm{~cm}$ to

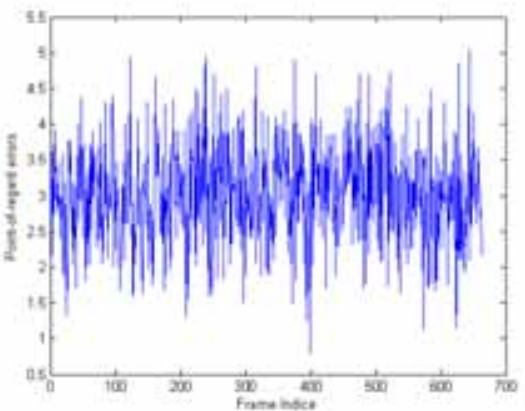

Figure 9: Point-of-regard error of the marker 1

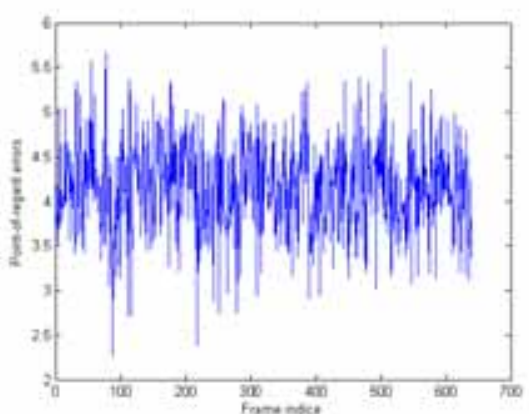

Figure 10: Point-of-regard error of the marker 2

the screen, the range of the pupil movement is then about 16 pixels. Let's consider our method of calculating the gaze direction (figure 13). In the figure, $|l| \in[0,8]$ pixels, $r \approx 20$ pixels. The smallest level unit of $l$ is $|\Delta l|=1$ pixel. Then,

$$
\begin{aligned}
\theta & =\arcsin l / r \\
\Delta \theta & \approx \frac{\partial \theta}{\partial l} \Delta l+\frac{\partial \theta}{\partial r} \Delta r \\
& =\frac{1}{r \sqrt{1-(l / r)^{2}}} \Delta l-\frac{1}{r^{2} \sqrt{1-(l / r)^{2}}} \Delta r
\end{aligned}
$$

Supose $\Delta r=0$, then for $|l| \in[0,8]$ pixel, $|\Delta \theta|=\left[2.86^{\circ}, 3.41^{\circ}\right]$. Thus, the smallest unit of $\theta$, i.e. the resolution of gaze direction is about $3.2^{\circ}$. If the iris detection has a small error of 1 pixel, it will generate a tracking error of about $3.2^{\circ}$ of gaze direction. Therefore, it is hard to determine the gaze in a 3D scene accurately with such low resolution, nevertheless in our use we only need to determine a region in the screen.

\section{Conclusion}

In this paper, a real-time implementation of a new method to estimate gaze direction using stereo vision is proposed. The motivation of our approach is to develop a low-cost device allowing to estimate the eye gaze robustly in real-time and with satisfactory accuracy. The first originality of our algorithm is the use of the target in order to recognize, localize and finally to quickly track the head of all persons. Also by using the described "oculometric calibaration" described, we were able to discard other features detection and obtain a proper eyeball center. Because of this, our method is adapted to 


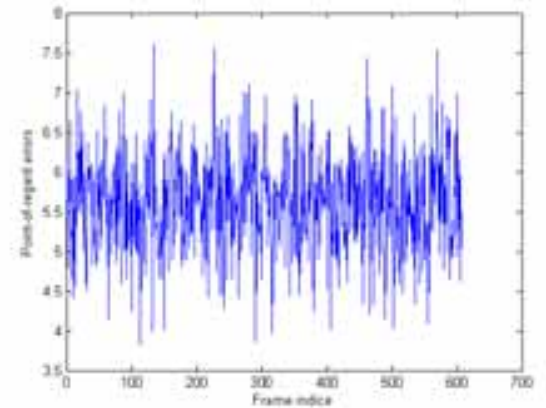

Figure 11: Point-of-regard error of the marker 3

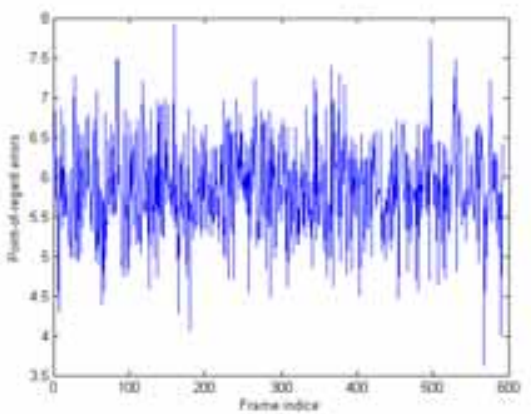

Figure 12: Point-of-regard error of the marker 4

different person and the accuracy of the visual direction should not be affected by the change of head pose. In summary, we developed a low-coast system (only two webcams) which observes a person without giving him/her any discomfort and shortly, this system will be applied for the collaborative tasks survey.

Currently, our algorithm is coded with Matlab and we got a satisfactory execution speed $(13 \mathrm{~Hz})$, in our future work; we will increase processing speed of the system by using $\mathrm{C}++$. We also aim to improve the accuracy by using high resolution camera and other image processing tools to detect the pupil (not the iris center).

\section{References}

ANDERS, G. 2001. Pilots attention allocation during approach and landingeye- and head-tracking research in an a330 full flight simulator. In International Symposium on Aviation Psychology (ISAP).

BAKer, M., Lund, K., And Quignard, M. 2002. Multirepresentationnal argumentative interactions : the case of computer-mediated communication in cooperative learning situation. In Proceedings of the Fifth International Conference of the International Society for the Study of Argumentation (ISSA'02).

Baluja, S., And Pomerleau, D. 1994. Non-intrusive gazetracking using artificial neural networks. In Neural Information Processing Systems, Morgan Kaufman Publishers.

Cho, Y., And NeUmann, U. 1998. Multi-ring color fiducial systems for scalable fiducial tracking augmented reality. In Proceedings of the Virtual Reality Annual International Symposium (VRAIS98), 212.

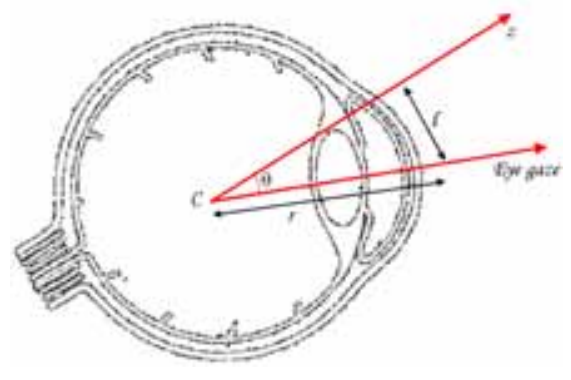

Figure 13: Image resolution problem

Collewijn, H. 1999. Multi-representationnal argumentative interactions : the case of computer-mediated communication in cooperative learning situation. In H. S. Carpenter and J.G.Robson [Eds.], Vision Research: A practical Guide to Laboratory Methods, 245-285.

Corbel, A., Jaillon, P., Serpaggi, X., BAKer, M., QuigNARD, M., Lund, K., AND Sjourn, A. 2003. A drew: Un outil internet pour crer des situations d'apprentissage cooprant. In Environnements Informatiques pour l'Apprentissage Humain (EIAH 2003).

Duchowski, A. T. 2002. A breadth-first survey of eye tracking applications. In IEICE Behavior Research Methods, Instruments, and Computers.

FIALA, M. 2005. Artag, a fiducial marker system using digital techniques. In Proceedings of the 2005 IEEE Computer Society Conference on Computer Vision and Pattern Recognition (CVPRO5), Volume 2, 590-596.

Glenstrup, A., And Nielson, T. 1995. Eye controlled media : Prensent and future state. In Masters thesis, University of Copenhagen.

Henderson, J. M., And Hollingworth, A. 1998. Eye movements during scene viewing: An overview. In G.Underwood (Ed.), Eye Guidance in Reading and Scene Perception.

Kato, H., And Billinghurst, M. 1999. Marker tracking and hmd calibration for a video-based augmented reality conferencing system. In Proceedings of the 2nd IEEE and ACM International Workshop on Augmented Reality (IWAR99), 85-92.

Kato, H., Billinghurst, M., Poupyrev, I., Imamoto, K., AND TACHIBANA, K. 2000. Virtual object manipulation on a table-top ar environment. In Proceedings of the International Symposium on Augmented Reality (ISAR 2000), 111-119.

Kim, K., AND RAMAKRISHNA, R. 1999. Vision-based eye-gaze tracking for human computer interface.. In International Conference on Systems, Man, and Cybernetics, 324-329.

Matsumoto, Y., And Zelinsky, A. 2000. An algorithm for real-time stereo vision implementation of head pose and gaze direction measurement. In International Conference on Automatic Face and Gesture Recognition, 499-504.

Matsumoto, Y., And Zelinsky, A. 2000. An algorithm for real-time stereo vision implmentation of head pose and gaze direcetion measurement. In International Conference on Automatic Face and Gesture Recognition, 499-504.

NAIMARK, L., AND Foxlin, E. 2002. Circular data matrix fiducial system and robust image processing for a wearable vision- 
inertial self-tracker. In Proceedings of the International Symposium on Mixed and Augmented Reality (ISMAR02), 27-36.

Pastoor, S., LiU, J., AND Renault, S. 1999. An experimental multimedia system allowing 3-d visualization and eyecontrolled interaction without user-worn devices. In IEEE Trans. Multimedia, 1(1), 41-52.

Perona, P., AND MALIK, J. 1990. Scale-space and edge detection using anisotropic diffusion. In IEEE Transactions on pattern and machine intelligence. Vol 12. NO. 7, 629-639.

Pomplun, M., VelichKovsky, B., AND Ritter, H. 1994. An artificial neural network for high precision eye movement tracking. In B. Nebel and L. Drescher-Fischer (Eds.), Lectures Notes in Artificial Interlligence, Springer Verlag.

Reingold, E. M., Charness, N., Pomplun, M., And STAMPE, D. M. 2002. Visual span in expert chess players: Evidence from eye movements. In Psychological Science.

Rekimoto, J., AND AyatsukA, Y. 2000. Cybercode : designing augmented reality environments with visual tags. In Proceedings of DARE 2000 on Designing augmented reality environments (DAREO0), 1-10.

Reкiмото, J. 1998. Matrix : A realtime object identification and registration method for augmented reality. In Proceedings of the Third Asian Pacific Computer and Human Interaction (APCHI98), 6368.

SibERT, L. E., AND JACOB, R. J. 2000. Evaluation of eye gaze interaction. In Human Factors in Computing Systems: CHI 2000 Conference Proceedings. ACM Press.

STIEFELHAGEN, R., AND YANG, J. 1997. Gaze tracking for multimodal human-computer interaction. In International Conference on Acoustics, Speech, and Signal Processing, 2617-2620.

TALMI, K., AND LIU, J. 1999. Eye and gaze tracking for visually controlled interactive stereoscopic displays. In Signal Processing: Image Communication 14., 799-810.

TOYAMA, K. 1998. Look, ma . no hands!. hands-free cursor control with real-time $3 \mathrm{~d}$ face tracking. In Workshop on Perceptual User Interfaces.

VertegaAl, R. 1999. The gaze groupware system: Mediating joint attention in multiparty communication and collaboration. In Proceedings of ACM CHI'99 Conference on Human Factors in Computing Systems.

Wang, J. G., Sung, E., And Venkateswarlu, R. 2003. A eye gaze estimation from a single image of one eye. In Proceedings of the Ninth IEEE International Conference on Computer Vision (ICCV 2003) 2-Volume.

Wu, H., Chen, Q., And WadA, T. 2005. Visual direction estimation from a monocular image. In IEICE Trans. Inf. and Syst., Vol. E88D, No.10., 2277-2285.

ZHANG, Z. 1999. Flexible camera calibration by viewing a plane from unknown orientations. In IEEE International Conference on Computer Vision.

Zhu, J., AND YANG, J. 2002. Subpixel eye gaze tracking. In IEEE International Conference on Automatic Face and Gesture Recognition. 